\title{
LA ENSEÑANZA DE LA FILOSOFÍ́ DE LA CIENCIA EN EL PERÚ EN LA ACTUALIDAD
}

\author{
THE TEACHING OF THE PHILOSOPHY OF SCIENCE IN PERÚ TODAY \\ (D) Manuel Abraham Paz y Miño Conde ${ }^{1^{*}}$ \\ mpaz@ulima.edu.pe \\ ${ }^{1}$ Universidad de Lima, Lima, Perú
}

*Correspondencia: Manuel Abraham Paz y Miño Conde. Email: mpaz@ulima.edu.pe

Recibido: 15.04.19 | Aprobado: 01.06.19

\section{RESUMEN}

En este artículo mencionaremos: a) los cursos de Epistemología, Filosofía de la Ciencia (y de la Tecnología), Teoría de la Ciencia e Introducción a la Ciencia que se enseñan en diversas universidades peruanas tanto públicas como privadas a nivel del pre y post grado; b) los libros publicados, en el país, sobre la materia por docentes universitarios; c) diversos eventos académicos sobre la materia, organizados en las universidades; y d) algunos números de revistas dedicadas a la filosofía de la ciencia.

Palabras clave: Cursos, ciencia, epistemología, universidades peruanas.

\section{ABSTRACT}

In this article we will mention: a) the courses of Epistemology, Philosophy of Science (and Technology), Theory of Science and Introduction to Science that are taught in various Peruvian universities both public and private at the pre and post-graduate level; b) the books published, in the country, on the subject by university professors; c) various academic events on the subject organized in the universities; and d) some issues of journals dedicated to the philosophy of science.

Keywords: Courses, science, epistemology, Peruvian universities. 


\section{INTRODUCCIÓN}

Saber qué es la ciencia, sus funciones y su clasificación, diferenciar la ciencia de la filosofía, la religión y la pseudociencia, en qué consiste el método científico, una hipótesis, una teoría y una ley científica, entre otras cosas, es el propósito del curso universitario de Epistemología que, muchas veces, se llama Filosofía de la Ciencia (que abarca la ontología, gnoseología, la ética, etc. de la ciencia) en países de habla castellana como el nuestro. Es un conocimiento esencial que todo estudiante universitario y futuro profesional debe saber para interpretar la realidad de modo científico y saber diferenciar que es conocimiento científico y qué no lo es, qué es creencia, superstición o prejuicios. Hay diversas definiciones de lo que es y de lo que trata la filosofía de la ciencia:

"Philosophy of science" is the name given to that branch of philosophy that reflects on and critically analyzes science. As a discipline, it tries to understand the aims and methods of science, along with its principles, practices, and achievements. Philosophers try to provide precise answers to very broad questions about science, such as the question just raised about the nature of scientific explanation. (Salmon et al., 1992, p. 1). [S] e trata del examen filosófico de la ciencia: de sus problemas, métodos, técnicas, estructura lógica, resultados generales, etc. (Bunge, M., 1960, p. 63). La filosofía de la ciencia investiga el conocimiento científico y la práctica científica (Wikipedia: Filosofía de la ciencia).

En la actualidad hay toda una gama de cursos y docentes peruanos dedicados a la Filosofía de la ciencia (conocida en nuestro medio como epistemología y esta, a su vez, equivale al inglés epistemology que, según la tradición anglo-sajona, es entendida como teoría del conocimiento, que nosotros llamamos gnoseología), además de cursos muy relacionados y complementarios como Lógica y Metodología de la Investigación Científica.

En este escrito solo nos ocuparemos de mencionar: a) los cursos de Epistemología, Filosofía de la Ciencia (y de la Tecnología, de ser el caso) con sus diversas subespecializaciones según el área, Teoría de la Ciencia e Introducción a la Ciencia que se enseñan en diversas universidades peruanas tanto públicas como privadas a nivel del pre y post grado; b) los libros publicados sobre la materia por docentes universitarios de y en nuestro país; c) diversos eventos académicos sobre la materia, organizados en las universidades; y d) algunos números de revistas dedicadas a la filosofía de la ciencia.

\section{LA FILOSOFÍA DE LA CIENCIA EN UNIVERSIDADES PÚBLICAS}

\section{Universidad Nacional Mayor de San Marcos (UNMSM) de Lima}

\section{Cursos:}

- Introducción a la Ciencia en los Estudios Generales del Área de Humanidades, Ciencias Jurídicas y Sociales (desde el 2018).

- Epistemología de la Comunicación en la Escuela de Comunicación.

- Epistemología, Filosofía de la Biología, Filosofía de las Ciencias Naturales, Filosofía de las Ciencias Sociales, Filosofía de la Lógica, Filosofía de la Matemática, Filosofía de la Psicología, Filosofía del Derecho y Filosofía de la Educación en la Escuela de Filosofía de la Facultad de Letras y Ciencias Humanas. 
- Epistemología de las Ciencias Formales y Naturales en la Maestría de Filosofía con Mención en Epistemología de la Unidad de Posgrado (UP) de la misma Facultad.

- Seminario de Investigación en Epistemología A, B y C se dicta en el Doctorado de Filosofía de la mencionada UP.

- $\quad$ Epistemología en el Doctorado en Farmacia y Bioquímica.

- Filosofía de las Ciencias médicas en el Doctorado de Medicina de la Unidad de Posgrado de la misma especialidad.

- Epistemología en el pregrado la Escuela de Educación de la Facultad de Educación de la UNMSM.

Cabe anotar que hasta hace unos años se enseñaba el curso de Epistemología en todas las menciones de la Maestría en Educación de la Unidad de Posgrado de la Facultad del mismo nombre, permaneciendo el de Metodología de la Investigación Científica.

\section{Libros:}

Los escritos por el finado Julio Sanz Elguera (1987), los ya cesantes como catedráticos, pero aún vivos Francisco Miró Quesada C. (1976), y Juan Rivera Palomino (2009), y los aún activos José Carlos Ballón (1999), Marino Llanos (2009), Roberto Katayama (2017), Carlos Alvarado de Piérola (2005), Raymundo Casas Navarro y el jubilado Carlos Matta Rojas (2006), Óscar García Zárate (2011) todos, excepto Casas, del Departamento de Filosofía de la Facultad de Letras y Ciencias Humanas de la UNMSM, y también por supuesto, Luis Piscoya Hermoza (2009), docente de posgrado.

Además, libros por Arsenio Guzmán Jorquera (2005), Carlos Barriga (2011) y Alberto Vásquez Tasayco (2012), el primero ex docente de la UP de Educación y los dos restantes de la Escuela de Educación de la Facultad de Educación de la UNMSM.

\section{Eventos:}

- Viernes filosóficos organizados por el Departamento Filosofía de la UNMSM donde se han presentado conferencias de epistemología, entre otros.

- I Curso Internacional de Filosofía "Epistemología y Metodología de las Ciencias" (2007), II Curso Internacional de Filosofía "Lógica y Filosofía de la Lógica: Gottlob Frege" (2009), III Curso Internacional de Filosofía “De Galileo a Einstein" (2010), IV Curso Internacional de Filosofía "Ciencia y realidad" (2011), V Curso Internacional de Filosofía "La filosofía del humanismo en el pensamiento español" (2011), VI Curso Internacional de Filosofía "La Teoría de Darwin, su recepción crítica y vigencia científica y filosófica" (2012), y VII Curso Internacional de Filosofía: "El Impacto Filosófico de la Psicología Darwiniana" (2014) organizados por la Escuela y el Departamento de Filosofía.

- Seminario Internacional de Epistemología "Ciencia, complejidad y globalización" (2008), o el Ciclo "Filosofía, ciencia y sociedad después de Darwin" (2009) también organizados por tal Departamento. 
- 4 Seminarios Internacionales de Epistemología (2008 y 2009), 3 Coloquios Peruanos de Filosofía de las Ciencias (2005, 2007 y 2009), 2 Cursos Internacionales de Epistemología y Transdisciplinariedad (2006 y 2009), y 2 Jornadas AmericanoEuropeas de Ciencia y Filosofía (2010) organizados por un grupo de estudiantes del pregrado de filosofía: Diaporein-Círculo Peruano de Investigaciones Filosóficas y Transdisciplinarias, siendo el Presidente de su Comité Directivo, el controversial y desaparecido Antonio Ramírez-Victorio,

- Simposio Sobre Historia de la Ciencia «Julio C. Sanz Elguera» (2017) organizado por la Asociación Peruana de Historia de la Ciencia (APHC), conformada por docentes preparados en la UNMSM.

- $\quad$ I y II Seminario "Ciencias, Pseudociencias y Terapias Pseudocientíficas" (2011 y 2012) organizado por la Unidad de Posgrado de la Facultad de Educación.

- III Seminario "Ciencias, Pseudociencias y Terapias Pseudocientíficas" (2015) organizado por el Centro Cultural de la UNMSM.

- $\quad$ IV Seminario "Ciencias, Pseudociencias y Terapias Pseudocientíficas" (2011 y 2012) organizado por el Departamento de Física de la UNMSM (2016).

- Los 4 seminarios coorganizados con la asociación Humanistas Racionalistas del Perú (HURA-PERÚ).

\section{Universidad Nacional Federico Villarreal (UNFV) de Lima}

\section{Cursos:}

- Epistemología en las Escuelas de Ciencias de la Comunicación, Filosofía, Psicología, Sociología.

- Epistemología de las Ciencias Sociales en la de Trabajo Social.

- Epistemología del Cambio en el Programa de Segunda Especialidad en Terapia Familiar Sistémica de la Unidad de Posgrado de la Facultad de Psicología.

\section{Eventos:}

El Departamento y la Escuela de Filosofía de la UNFV organizan los Jueves Filosóficos y la segunda a la fecha ha organizado 15 Fórums de Filosofía con temas que incluyen filosofía de la ciencia, como, en el primer caso, el Seminario Internacional por el Bicentenario del Naturalista Charles Darwin (2009) y mesas diversas en el segundo.

Universidad Nacional de Educación Enrique Guzmán y Valle (UNE)-La Cantuta de Chosica

\section{Cursos:}

- Epistemología en la mayoría de las menciones de la Maestría en Ciencia de la Educación de la Escuela de Posgrado (EP).

- Epistemología de la matemática en la Mención Didáctica de la Matemática de la mencionada Maestría. 
- Epistemología en Administración en la Maestría en Administración en la misma EP.

\section{Libros:}

Por Bladimiro Guevara (2003, 2015), ex docente de la EP de la UNE y Víctor Mazzi Huaycucho (2010) actual docente de la Facultad de Ciencias Sociales y Humanidades; además por Nicómedes Esteban Nieto, Julio Carhuaricra Meza y Moisés Esteban Nieto (2015) de la misma EP.

\section{Universidad Nacional de San Agustín (UNSA) de Arequipa}

\section{Cursos:}

- $\quad$ Epistemología general para diversas carreras.

- Epistemología General e Investigación filosófica del Derecho (3er año del pregrado de la Escuela de Filosofía).

- Seminario de Filosofía de las ciencias sociales e Investigación filosófica sobre Educación (4to año).

- $\quad$ Filosofía y Tecnología (anual).

- Filosofía de las ciencias formales y Seminario de filosofía de las ciencias naturales (5to. año).

\section{Libros:}

Oscar Barreda Tamayo (1997, 1999, 2004 y 2010) que enseñó en el pregrado de filosofía y ahora en el Escuela de Posgrado y publicó con Elita Oviedo Pinto (1999), también está Luis Salluca Quispe (2014, 2015).

\section{Eventos:}

En 1997, el Departamento de Filosofía organizó el I Curso Internacional de Postgrado: Epistemología de las ciencias y técnicas naturales y sociales" con del Dr. Mario Bunge, y recientemente el Congreso Internacional de Filosofía de la Ciencia y la Tecnología (2016) y acaba de publicar las respectivas Actas (2017).

La Oficina de Cultura de la UNSA organizó, en el 2011, el Curso Internacional de Postgrado "Filosofía de la Ciencia y Conocimiento del Universo" con el ahora fenecido Dr. Jesús Mosterín.

\section{Universidad Nacional de Trujillo (UNT)}

\section{Cursos:}

- Epistemología en la Escuela de Educación en el Nivel Inicial, Primaria y en la Escuela de Ciencias de la Comunicación.

- Epistemología y Sistematización Teórica en la Maestría en Educación, con mención en Epistemología y Teoría Educativa.

- Filosofía de la Ciencia en todos sus Doctorados. 


\section{Libros:}

Por Víctor Baltodano Azabache (2010) y Carlos Lázaro Arroyo (2010), docentes de la Escuela de Posgrado de la UNT.

\section{Universidad Nacional José Faustino Sánchez Carrión (UNJFSC) de Huacho}

\section{Cursos:}

- Filosofía de la Ciencia y de la Tecnología en el pregrado de Sociología, Ciencia de la Comunicación, Trabajo Social, Estadística e Informática, Biología, Matemática Aplicada, Ingeniería Agronómica, Ambiental, Industrias Alimentarias, Zootécnica, Industrial, de Sistemas, Informática y Electrónica, Metalúrgica, Química, etc.

- Filosofía, Ciencia y Tecnología en el pregrado de Física, Ingeniería Pesquera, y Acuícola, etc.

- Epistemología Educativa en la Maestría en Ciencias de la Gestión Educativa, en las menciones de Pedagogía, Motricidad Humana o Educación Ambiental de la Escuela de Posgrado.

\section{Libros:}

Jenner Ricardo Ramos Murphy (2008, 2009 y 2010) quien enseñó en la UNJFSC hasta el 2016.

\section{Universidad Nacional de San Cristóbal de Huamanga (UNSCH)}

\section{Cursos:}

- Epistemología de las Ciencias Sociales en la Maestría en Ciencias Sociales, con mención en Antropología en la Sección de Post Grado de la Facultad de Ciencias Sociales.

- Bases Epistemológicas en la Investigación Educativa en el Doctorado en Educación en la Sección de Post Grado de la Facultad de Ciencias de la Educación.

Universidad Nacional de San Antonio Abad del Cusco (UNSAAC)

\section{Cursos:}

- Filosofía de la Ciencia para la mayoría de carreras, y Epistemología en el Doctorado de Derecho y en el de Administración.

Universidad Nacional de la Amazonía Peruana (UNAP) de Iquitos

\section{Cursos:}

- Epistemología para el pregrado de Educación, Especialidad de Filosofía y Psicopedagogía.

- Epistemología y Gnoseología para la Especialidad de CCSS.

- Epistemología Educativa en el Doctorado de Educación.

- $\quad$ Epistemología Jurídica en el Doctorado de Derecho.

- Epistemología en el Doctorado en Ciencias Ambientales. 


\section{Universidad Nacional del Altiplano - Puno (UNAP)}

\section{Cursos:}

- Epistemología para el pregrado de Educación Inicial y Educación Secundaria, especialidad: Lengua, Literatura, Psicología, y Filosofía.

- $\quad$ Epistemología de las Ciencias Sociales para el pregrado de Sociología.

- Epistemología de la Investigación Antropológica para el pregrado de Antropología.

- $\quad$ Filosofía del Derecho y Epistemología Jurídica para el pregrado de Derecho.

Universidad Nacional "San Luis Gonzaga" de Ica (UNICA)

\section{Cursos:}

- Introducción a la Epistemología para el pregrado de Educación, mención: Filosofía, Psicología y Ciencias Sociales.

- Epistemología y Metodología de la Investigación científica en las Maestrías en Administración, menciones: Gestión Empresarial, y Gestión Pública; Agronomía, menciones: Producción Agrícola, y Agronegocios; Ciencias de la Comunicación; Economía, Educación, menciones: Administración y Planificación de la Educación, y Gestión Educativa, Derecho, menciones: Civil y Comercial, y Ciencias Penales; Ingeniería Civil, mención: Gestión y Gerencia de la Construcción; Ingeniería Mecánica; Ingeniería Química; Ingeniería de Sistemas; Salud Pública; Ingeniería de Sistemas.

- Epistemología en las Maestrías en Ingeniería; Farmacia y Bioquímica; Química Farmacéutica.

- Epistemología e investigación científica en el Doctorado en Salud Pública.

- Seminario y Epistemología del Derecho en el Doctorado en Derecho.

- Epistemología en los Doctorados en Educación, y Farmacia y Bioquímica.

\section{LA EPISTEMOLOGÍA EN LAS UNIVERSIDADES PRIVADAS}

Pontificia Universidad Católica del Perú (PUCP) de Lima

\section{Cursos:}

- $\quad$ Epistemología en Estudios Generales (EEGG) de Letras, Pregrado de Filosofía.

- Filosofía de la ciencia en Pregrado de Filosofía.

- Ciencia y Filosofía en EEGG de Ciencias.

- Filosofía de las Matemáticas en Pregrado de Filosofía.

- $\quad$ Filosofía de la Mente en Pregrado de Filosofía.

Universidad Inca Garcilaso de la Vega (UIGV) de Lima

Cursos: 
- Epistemología para la Carrera Profesional de Educación Nivel Inicial y la de Primaria.

- Epistemología para las Maestrías de Contabilidad, Derecho Administrativo, Derecho Civil y Comercial, Desarrollo Organizacional y Alta Dirección, Estomatología, Gerencia de Servicios de Salud, Gestión Educativa, Gobierno y Gestión Pública, Investigación y Docencia Universitaria, Política, Gestión y Derecho Ambiental, Psicología del Niño y el Adolescente, Salud Pública, etc.

Filosofía de la Ciencia en los Doctorados de Administración, Contabilidad, Derecho, Economía, Educación, Salud Pública.

- Seminario de Teoría de la Ciencia en el Doctorado de Medio Ambiente y Desarrollo Sostenible.

\section{Libros por:}

Roberto Katayama Omura y Víctor Pulido Capurro (2017), docentes de la Escuela de Posgrado de la UIGV.

\section{Eventos:}

- I Curso Internacional: Vigencia de la Filosofía (1996), el IV Curso Internacional: Una Filosofía Realista para el Nuevo Milenio (2001) y el Curso Internacional: Filosofía Política (2009), con el Dr. Mario Bunge de Argentina a quien le ha publicado diversos libros (2009, 2012, 20122, 2015, 20152).

- Curso Internacional Naturaleza y Cultura Humana: Del Genoma a Internet (2010), con el Dr. Jesús Mosterín de España a quien también le ha publicado diversos libros (2009, 2012, 20122, 2015, 20152).

\section{Universidad Católica Sedes Sapientiae (UCSS) de Lima}

\section{Cursos:}

Epistemología en el pregrado de Educación Inicial, Educación Primaria, Educación Especial, Educación Secundaria, especialidad de Filosofía y Religión, y Educación Secundaria, especialidad de Lengua Inglesa, Psicología.

\section{Libros por:}

Paolo Musso (2012), docente de la Universidad de Insubria (Italia) y profesor visitante en la UCSS donde enseña el curso de Epistemología en la Facultad de Ciencias de la Educación y Humanidades desde el 2005.

\section{Universidad Antonio Ruiz de Montoya (UARM) de Lima}

\section{Curso:}

Filosofía y Ciencia para la Carrera de Filosofía.

Universidad Facultad de Teología Pontificia y Civil de Lima (FTPCL)

\section{Curso:}

Filosofía de la Ciencia para las Carreras de Filosofía y Teología. 


\section{Universidad de San Martín de Porres (USMP)}

Curso:

Epistemología en el Doctorado de Medicina.

Libro:

Por Bernardo Rea Ravello (1993).

\section{Universidad Ricardo Palma (URP) de Lima}

\section{Curso:}

Epistemología en la Escuela de Psicología, que tiene como prerrequisito el de Lógica, curso que hasta no hace mucho se enseñaba en la mayoría de carreras de ahí.

\section{Universidad César Vallejo (UCV)}

Cursos:

- Epistemología en el Doctorado en Educación

- Epistemología de la Psicología en el Doctorado en Psicología

- Epistemología de la Comunicación en el Doctorado en Comunicación Social

Libro:

Por Guillermo Príncipe Cotillo (2016).

\section{Universidad San Ignacio de Loyola (USIL) de Lima}

\section{Cursos:}

- Filosofía de la Ciencia en las carreras de Diseño y Gestión de Marca, Multimedia y Fotografía Publicitaria.

- Fundamentos Epistemológicos de la Psicología en la de Psicología.

- Epistemología de la Educación en la Maestría y Doctorado de Educación.

- Epistemología Jurídica en el Doctorado de Derecho.

Universidad de Ciencias y Humanidades (UCH)

\section{Cursos:}

- Filosofía, Ciencia y Tecnología en el pregrado de todas sus carreras, a saber: Educación Primaria e Interculturalidad, Ingeniería de Sistemas e Informática, Ingeniería Electrónica con mención en Telecomunicaciones, Enfermería, Contabilidad con mención en Finanzas.

\section{Eventos:}

- Ciclo de Conferencias Ciencia y Pseudociencia: Una Introducción a la Epistemología (2010) por miembros de la Asociación Peruana de Historia de la Ciencia.

\section{Universidad Norbert Wiener (UWIENER)}

\section{Cursos:}

Investigación Jurídica y Epistemología en la Maestría en Derechos Fundamentales. 
Universidad Privada Antenor Orrego (UPAO) de Trujillo

\section{Cursos:}

Filosofía de la Ciencia se enseña tanto a las carreras de ciencias como de letras.

\section{Universidad Católica San Pablo (UCSP) de Arequipa}

\section{Cursos:}

- Epistemología de la Educación en el pregrado de Educación, especialidades en Educación Inicial, y Educación Primaria.

\section{REVISTAS SOBRE FILOSOFÍA DE LA CIENCIA}

Actualmente, no existe en el Perú una publicación periódica exclusivamente dedicada a la Filosofía de la Ciencia o la Epistemología, actividad que les correspondería a las sociedades académicas dedicadas al tema como la aún activa Asociación Peruana de Historia de la Ciencia, fundada en el 2013 por profesionales sanmarquinos, a diferencia de la ya descontinuada Sociedad Peruana de Epistemología, fundada en 1994 con docentes y alumnos del posgrado de filosofía y cuyo primer y único presidente fue el Dr. Luis Adolfo Piscoya Hermoza.

Lo que si ha habido es una serie de revistas de filosofía que han publicado algunos artículos de la especialidad como, por ejemplo, la desaparecida Yachay o su sucesora Sullul publicada por algunos docentes del Departamento de Filosofía, UNMSM; o Reflexión y Crítica. Revista de Filosofía, publicación oficial del mismo Departamento; Letras de la Facultad de Letras y CCHH de la UNMSM; Magistri et Doctores que la Escuela de Post Grado de la UNMSM publicó entre 1993 y 2006, Areté del Departamento de Humanidades de la PUCP, Analítica del Centro de Estudios de Filosofía AnalíticaCESFIA, etc.

Sin embargo, cabe mencionar que el nro. 3 de la Revista Peruana de Filosofía Aplicada (RPFA) de la cual el autor de estas líneas es Director, fue dedicado a la Filosofía de la Ciencia. Además, los 4 números impresos de la ya descontinuada revista Neo-Skepsis fueron dedicados a desenmascarar diversas pseudociencias.

\section{CONCLUSIONES}

Hemos podido ver que hay un interés variado por enseñar cursos de Epistemología o Filosofía de la Ciencia con sus variables y especializaciones en las universidades públicas o privadas, confesionales o no.

En unas universidades se enseña en casi todas las carreras a nivel de pregrado, en otras solo a unas cuantas, y en unas no enseñan tales cursos en el pregrado. Otras solo en la maestría o el doctorado o ningún curso de la especialidad en el posgrado.

Entonces no todos los egresados tienen la misma formación y eso explicaría por qué no pocos de ellos ni siquiera sabe de qué se ocupa la ciencia, cuál es su objeto de estudio, qué tipos de ciencia hay, etc., o peor, que algunos profesionales a pesar de su formación universitaria practiquen alguna pseudociencia o pseudotecnología.

\section{REFERENCIAS BIBLIOGRÁFICAS}

Alvarado, Carlos (2005). Epistemología. Lima: Mantaro. 
Lázaro Arroyo, Carlos (2010). Epistemología de la Pedagogía. Una evaluación dialéctica. Trujillo: Ediciones Nuevo Educar.

Ballón, José Carlos (1999). De la física moderna a la física contemporánea. Un cambio en nuestro paradigma de ciencia. Lima: CONCYTEC.

Baltodano Azabache, Víctor (2010). Filosofía de las ciencias para la creación de conocimientos. Lima: San Marcos.

Barreda Tamayo, Oscar (1997). Selección de textos de Mario Bunge Arequipa: UNSA para el Primer curso internacional de postgrado a cargo del Dr. Mario Bunge: Epistemología de las ciencias y técnicas naturales y sociales.

Barreda Tamayo, Oscar y Oviedo Pinto, Elita (1999). Epistemología General. Antología. 2a. ed. 2001, 3a. ed. 2002 Arequipa: UNSA.

Barreda Tamayo, Oscar (2004). Epistemología de la Matemática. Antología. 2a . ed. 2006, $3^{\text {a }}$ ed. 2007. Arequipa: UNSA Escuela de postgrado Matemática.

Barreda Tamayo, Oscar (2010). Epistemología de las Ciencias Sociales. 2da. ed. 2012 Arequipa: UCSM Estudios a distancia.

Barriga Hernández, Carlos (editor) (2011). Introducción a la epistemología. Lima: UNMSM, Facultad de Educación, Programa de Complementación Pedagógica.

Beorlegui, Carlos (2008). Historia del pensamiento filosófico latinoamericano. Bilbao: Universidad de Deusto.

Bunge, Mario (1960). La ciencia. Su método y su filosofía. Ediciones Siglo Veinte, Buenos Aires.

Bunge, Mario (2009). Estrategias de la investigación científica. Lima: Fondo Editorial UIGV.

Bunge, Mario (2012). Diálogos urticantes. Lima: Fondo Editorial UIGV.

Bunge, Mario (20122). Filosofía de la tecnología y otros ensayos. Lima: Fondo Editorial UIGV.

Bunge, Mario (2015). Vigencia de la filosofía. Lima: Fondo Editorial UIGV.

Bunge, Mario (20152). ¿Qué es filosofar científicamente? Lima: Fondo Editorial UIGV.

Casas Navarro, Raymundo y Carlos Matta Rojas (2006). El método científico. Lima: Mantaro.

Cordero, A. (2009) "Philosophy of Science", in A Companion to Latin American Philosophy (eds S. Nuccetelli, O. Schutte and O. Bueno), Wiley-Blackwell, Oxford, UK.

Cordero, Alberto (2015) "Philosophy of Science in Latin America", in The Stanford

Encyclopedia of Philosophy, Edward N. Zalta (ed.), URL = http://plato.stanford.edu/archives/win2015/entries/phil-science-latin-america/ 
Esteban Nieto, Nicomedes; Julio Carhuaricra Meza y Moisés Esteban Nieto (2015). Antología de la Epistemología. Lima: UNE \&amp; Cerro de Pasco: UDAC.

García Zárate, Óscar Augusto (2011). Ciencia, verdad y filosofía, Lima, CEPREDIMUniversidad Nacional Mayor de San Marcos.

Gracia, Jorge J. E., Eduardo Rabossi, Enrique Villanueva \&amp; Marcelo Dascal (eds.) (1984). Philosophical Analysis In Latin America. Dordrecht: D. Reidel Publishing Company.

Guevara Gálvez Bladimiro (2002). Curso básico de epistemología: Filosofía y teoría de la ciencia. Lima.

Guevara Gálvez Bladimiro (2003). Epistemología de la Investigación. Lima: Ediciones Pensamiento y Acción.

Guevara Gálvez Bladimiro (2015). Educología: Epistemología de la Educación. Lima: Ediciones - Pensamiento y Acción.

Guzmán Jorquera, Arsenio (comp.) (2005). Epistemología. Lima: UPG-Facultad de Educación, UNMSM.

Katayama Omura, Roberto, y Pulido Capurro, Víctor (2017). Epistemología. Fondo Editorial de la UIGV.

Llanos Villajuán, Marino (2009). Epistemología de las Ciencias Sociales. Lima: Fondo Editorial de la UNMSM.

Mazzi Huaycucho, Víctor (2010). Epistemología. Selección de lecturas. Lima: Editorial San Marcos.

Miró Quesada C., Francisco (1976). Filosofía de las matemáticas. Lima: Ediciones Previa.

Mosterín, Jesús (2010). Naturaleza, vida y cultura. Lima: Fondo Editorial UIGV.

Mosterín, Jesús (2010). Diálogo y Debate. Lima: Fondo Editorial UIGV.

Mosterín, Jesús (2011). Filosofía y Ciencia. Un continuo. Lima: Fondo Editorial UIGV.

Mosterín, Jesús. Epistemología y racionalidad. Lima: Fondo Editorial UIGV, 2011.

Musso, Paolo (2012). Formas de la epistemología contemporánea. Entre realismo y antirealismo. Lima: Fondo Editorial UCSS.

Neo-Skepsis \# 1: Pseudociencias. Lima: Ediciones RPFA, s/f. Versión-e: http://www.reocities.com/cipsiperu/neo1.htm.

Neo-Skepsis \# 2: El Fenomeno Ovni. Lima: Ediciones RPFA, s/f. Versión-e: http://www.reocities.com/cipsiperu/neo2.htm.

Neo-Skepsis \# 3: Lo Paranormal y la Parapsicología. Lima: Ediciones RPFA, s/f. Versión-e: http://www.reocities.com/cipsiperu/neo3.htm.

Neo-Skepsis \# 4: Psicología y Pseudociencia. Lima: Ediciones RPFA, s/f. Versión-e: http://www.reocities.com/cipsiperu/neo4.htm. 
Piscoya Hermoza, Luis (2009). Tópicos en epistemología. Lima: Fondo Editorial UIGV, $2^{\mathrm{a}}$. ed.

Príncipe Cotillo, Guillermo (2016). Investigación Científica. Teoría y metodología. Lima: Universidad César Vallejo.

Ramos Murphy, Jenner (2008). La Hipótesis. Lima: s/n.

Ramos Murphy, Jenner (2009). El problema científico. Lima: s/n.

Ramos Murphy, Jenner Ricardo (2010). El Método Científico. Lima: s/n.

Rea Ravello, Bernardo (1993). Filosofía de la Ciencia. Lima: Amaru Editores.

Revista Peruana de Filosofía Aplicada (RPFA) Nro 3, Filosofía de la Ciencia. Lima:

Ediciones RPFA, s/f. Versión-e: http://filosofia-aplicada.humanists.net/res3.htm.

Rivera Palomino, Juan (2009). Epistemología Histórica, investigación y docencia. Lima: Concytec.

Salmon, Merrilee H., John Earman, Clark Glymour, James G. Lennox, Peter Machamer, J. E. McGuire, John D. Norton, Wesley C. Salmon, Kenneth F. Schaffner: Introduction to the philosophy of Science (1999). Indianapolis: Hackett Publishing Company, Inc., reimp.

Salluca Quispe, Luis (2014). Introducción a la lógica simbólica. Arequipa. 2008, 2da. Edición.

Salluca Quispe, Luis (1987). Introducción a la epistemología. Arequipa. 2015. Sanz, Julio. Introducción a la ciencia. Lima: Editorial Mantaro.

Vásquez Tasayco, Alberto (2012). El ABC de la epistemología. Análisis de la Ciencia. Lima: Fondo Editorial de la UNMSM.

Wikipedia: Filosofía de la ciencia. Recuperado de https://es.wikipedia.org/wiki/Filosof\%C3\%ADa_de_la_ciencia. actualidad. Puriq, 1(01), 12-24. https://doi.org/10.37073/puriq.1.01.7 